\title{
Crystal Structure of MJ0684 from Methanococcus jannaschii, a Novel Archaeal Homolog of Kynurenine Aminotransferase
}

\author{
Jin Kuk Yang \\ Department of Chemistry; Soongsil Lniversity Seoul 156-743, Korea. E-mail: jinkukvangassuackr \\ Recerved September 30, 2007
}

\begin{abstract}
MJ0684 from Methanococcus jannaschii is a hypothetical protein belonging to the subfamily I $\gamma$ of amino acid aminotransferases. In the present study the crystal structure of MJ068+ has been determined at $2.2 \AA$ resolution. It reveals that MJ0684 has an overall structure similar to subfamily I $\gamma$ aminotransferases and its active site architecture is most similar to that of kynurenine aminotransferases among several kinds of aminotransferases in the subfamily $\mathrm{I} \gamma$. It has two hydrophobic active site residues conserved in the kñurenine aminotransferases for recognizing hỵdrophobic substrates. In addition. the absence of any basic residue for recognizing the side chain carboxylic group of the aspartate in the active site nules out the possibility that MJ068+ would act as an aspartate aminotransferase. These structural observations collectively imply that MJ068+ is a novel archaeal homolog of the subfamily I $\gamma$ kynurenine aminotransferase.
\end{abstract}

Key Words : Amino acid aminotransferase, Kynurenine aninotransferase, MJ0684

\section{Introduction}

Pyridoxal 5'-phosphate (PLP)-dependent amino acid aminotransferase (AT) is a homodimeric enzyme which catalyzes the reversible transfer of an $\alpha$-amino group from an $\alpha$-amino acid to an $\alpha$-keto acid. ${ }^{\prime}$ ATs have been classified into four families. I to IV based on the homology relationships in the primary sequence." Family I. the most extensively studied one can be further divided into seven subfamilies: $\mathrm{I} \alpha, \mathrm{I} \beta \mathrm{I} \gamma$. and others. ${ }^{3}$ Subfamily Ia mostly consists of aspartate aminotranserases (AspATs) and aromatic amino acid aminotransferases (AroATs). Subfamily I $\beta$ includes histidine aminotransferases. some of which shows the activity toward aromatic amino acids as well as histidine. Subfamily I $\gamma$ includes AroATs such as kynurenine AT (KỵnAT) and tyrosine AT (TyrrAT). and some set of AspATs. ${ }^{3}$

Structural studies on a number of ATs have presented comprehensive observations on their various strategies for recognition of various amino acids as substrates. For example. subfamily I $\alpha$ AspATs from $E$. coli ${ }^{4}$ chicken ${ }^{56}$ pig. $^{7}$ and yeast ${ }^{\S}$ have a conserved arginine residue to recognize the side chain carboxylic group of the substrate aspartate (R292* in pig cytosolic AspAT. for instance: asterisk denotes the residue from the other subunit in dimer). In contrast. AspATs in the subfamily I $\gamma$ don't have the arginine residue in the corresponding position in the primary sequence, and instead use a lysine residue (K101 in AspAT from Thermis thermophihis HB8, for instance) for the same purpose. ${ }^{9}$

MJ0684 is a 370 -residue protein encoded by the Mij0684 (aspB2) gene from Methanococcus jannaschii. Sequence homology analysis using BLAST ${ }^{10}$ indicates that MJ0684 is a member of AT subfamily I $\gamma$. Among the subfamily I $\gamma$ members. it shows the highest sequence similarity to AspATs (34-41\% identity in 330-370 residues overlap). and then next to AroATs such as KynATs and TyrATs (25-30\% identity in 280-300 residues overlap). So BLAST results suggest that MJ0684 is most likely an AspAT in the subfamily I $\gamma$. However. MJ0684 lacks the otherwise conserved ly sine residue that plays a critical role in recognizing the side chain carboxylic group of the aspartate as a putative substrate. Thus. it may prefer other substrates with hydrophobic character such as kynurenine. tyrosine. phenylalanine and tryptophan rather than the aspartate, or it may have a different active site basic residue playing the same role of K101 of the AspAT from Thermis thermophilus HB8. MJ0684 structure in comparison with previously reported structures of ATs may be able to present some novel structural observations to answer this puzzling question.

Here I report the crystal structure of MJ0684 in complex with the cofactor PLP at $2.2 \AA$ resolution. Its overall structure is more similar to AspAT than AroATs such as KynAT and TyrAT. but the details of the active site architecture imply that it may prefer hydrophobic amino acids as substrates. There is no basic residue in the active site for recognizing the side chain carboxylate of the putative substrate aspartate. and there are found the two hydrophobic residues that are conserved in KynATs for recognizing hydrophobic amino acids as substrates

\section{Methods}

Protein expression, purification, crystallization and data collection from a native crystal have been described previously. "Molecular replacement calculation tried right after the native data collection was not successful. so soon $\mathrm{Hg}$ derivative crystal was prepared by adding $0.2 \mathrm{uL}$ of $1 \mathrm{mM}$ $\mathrm{Hg}(\mathrm{OAc})_{2}$ into $4 \mathrm{uL}$ of the hanging drop. After soaking for 2 hrs in this condition, the crystal was mounted and $X$-ray diffraction data were collected in the same way used for the native cry stal described previously. "The native data set was collected to $2.2 \mathrm{~A}$ and the derivative to $2.5 \mathrm{~A}$. The structure 
was solved by the single isomorphous replacement with anomalous scattering (SIRAS) approach. Total $+\mathrm{Hg}$ sites were located using SOLVE, ${ }^{12}$ and the phases were refined using SHARP. ${ }^{13}$ After density modification with SOLOMON, ${ }^{1+}$ an interpretable electron-density map was obtained for the space group $\mathrm{P}_{3} 2,2$ anong two enantiomeric space groups. Model building and refinement were completed using the programis $\mathrm{O}^{15}$ and CNS. ${ }^{16}$ Atomic coordinates and structure factors were deposited in Protein Data Bank under an accession code $2 Z 61$.

\section{Results and Discussion}

Crystal structure of MJ0684 has been determined and refined to $\mathrm{R}_{\text {free }}$ of $23.5 \%$ at a resolution of $2.2 \AA$. Asymmetric unit contains a single molecule of MJ0684 with a PLP covalently linked to Lys 222 and 87 water molecules. The functional dimer can be generated by crystallographic two-fold synmetry operation. The monomer model contains 369 residues of the entire 370-residue-long chain except for the last residue $\mathrm{K} 370$. Ramachandran plot produced by PROCHECK ${ }^{17}$ shows that all the residues except for only one residue, Ile253, are in the allowed region. Statistics for the data collection and the structure refinement are summarized in Table 1 . The overall architecture adopts the type I fold of PLP-dependent enzymes. which is characterized by an $N$-terminal arm and two domains. one small and the other large. as observed in other ATs except for the branched-chain anino acid ATs and D-amino acid ATs. ${ }^{18}$ The $N$-terminal arm (residues 1-11) consists of one 3 , helix and random coils. and the small domain (residues 12.43 and 269-369) contains four $\beta$-strands and four $\alpha$-helices. The large dontain (residues 44-268) adopts an $\alpha / \beta / \alpha$ structure in which a seven-stranded $\beta$-sheet is surrounded by nine $\alpha$-helices (Fig. 1A). MJ0684 formts a homodimer in a manner characteristic of PLP-dependent ATs with the type I fold (Fig. IB).

Sequence similarity search using BLAST ${ }^{1 \mathrm{C}}$ indicates that MJ0684 is a member of AT subfamily I $\gamma$ which includes AspAT. KynAT and TyrAT. ${ }^{\hat{3}}$ Among several kinds of ATs in the subfamily I $\%$. MJ0684 shows the highest sequence similarity to AspATs such as AspAT (TM1255) from Ther-
Table 1. Data Collection and Model Refinement

\begin{tabular}{|c|c|c|}
\hline & Native & $\mathrm{Hg}(\mathrm{OAc})_{2}$ \\
\hline Unit cell: $a=b, c(\AA)$ & $111.87,60.86$ & $112.01,60.91$ \\
\hline Resolution $(\mathcal{A})$ & $50.0-2.2$ & $50.0-2.5$ \\
\hline Completeness (\%) & $99.6(98.9)$ & $84.4(89.4)$ \\
\hline $\mathrm{I} / \sigma \mathrm{I})$ & $36.1(4.3)$ & $29.1(6.1)$ \\
\hline $\mathrm{R}_{\text {merat }}$ & $70(40.7)$ & $6.6(32.1)$ \\
\hline Observed reflections & 120,856 & 48.993 \\
\hline Unique retlections & 20,121 & 11,773 \\
\hline $\mathrm{R}_{\text {iyst }}{ }^{t} / \mathrm{R}_{\mathrm{bee}}{ }^{c}(\%)$ & $21.4 / 23.5$ & \\
\hline Runsd bond lengths $(A)$ & 0.008 & \\
\hline Rmsd bond angles $\left(^{\circ}\right)$ & 1.85 & \\
\hline Average B-factor & 39.6 & \\
\hline Protein atoms & 39.2 & \\
\hline Cofactor (PLP) & 38.9 & \\
\hline Waters & 50.2 & \\
\hline
\end{tabular}

Values in parenthesis are for the outer resolution shell. " $\mathrm{R}_{\text {merge }}=\Sigma_{\mathrm{h}} \Sigma_{\mathrm{l}} \mathrm{I}\left(\mathrm{l}_{1}\right)_{1}$

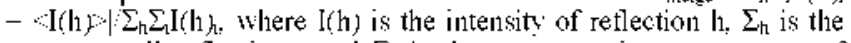
sum over all reflections, and $\Sigma_{j}$ is the sum over i measurements of

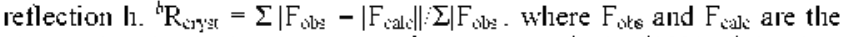
observed and calculated structure factor. respectively. " $10^{\circ} \%$ of the data was set aside for $R_{\text {trea }}$ calculation.

motoga maritima (tmAspAT: $41 \%$ identical in 362-residue overlap) $^{19}$ and AspAT from Thermus thermophitus HB8 (ttAspAT: 37\% identical in 369-residue overlap). ${ }^{5}$ In addition, MJ0684 also shows high sequence similarity to AroATs such as KynAT from mouse (33\% identical in 290-residue overlap) and TyrAT from Thponosoma cruzi (tcTyrAT: $29 \%$ identical in 342-residue overlap). ${ }^{\text {3i }}$ Structural similarity search using DALI server ${ }^{2]}$ also shows that MJ0684 is the most similar to AspATs such as ttAspAT ${ }^{9}$ (minsd $1.5 \AA$ in 363-residue overlap; PDB code (BJW), and then next to AroATs such as KynAT from Aedes aegap $i^{27}$ (misd $2.2 \AA$ in 347-residue overlap: PDB code IYIY) and tcTyrAT ${ }^{301}$ (nnsd $2.2 \mathrm{~A}$ in 356-residue overlap; PDB code (BW0). Although the similarity search in the primary sequence and tertiary structure commonly indicates MJ0684 is most likely a family I $\gamma$ AspAT, MJ0684 lacks the otherwise conserved lysine residue (for instance. K101 of ttAspAT) ${ }^{9}$ which recognizes the carboxylate in the side chain of the putative
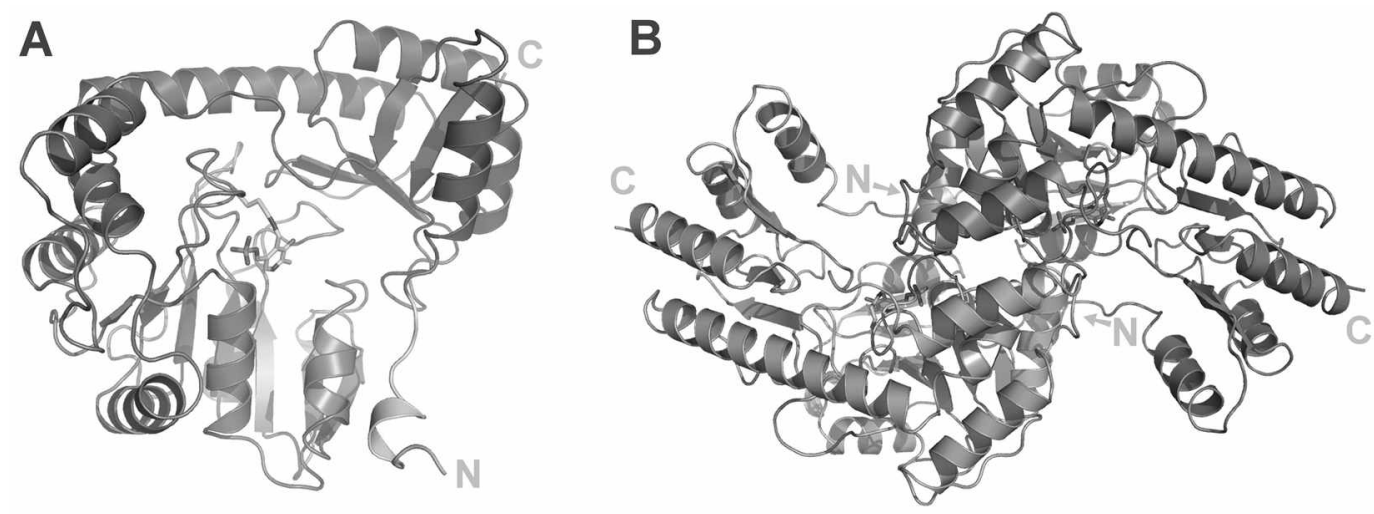

Figure 1. (A) Overall fold of a MJT684 monomer. PLP is shown in a stick model. N-terminal arm is colored in yellow, the small domain in purple, and the large domain in blue. (B) M.T0684 diner. One subunit in dimer is colored in purple and the other in blue. 

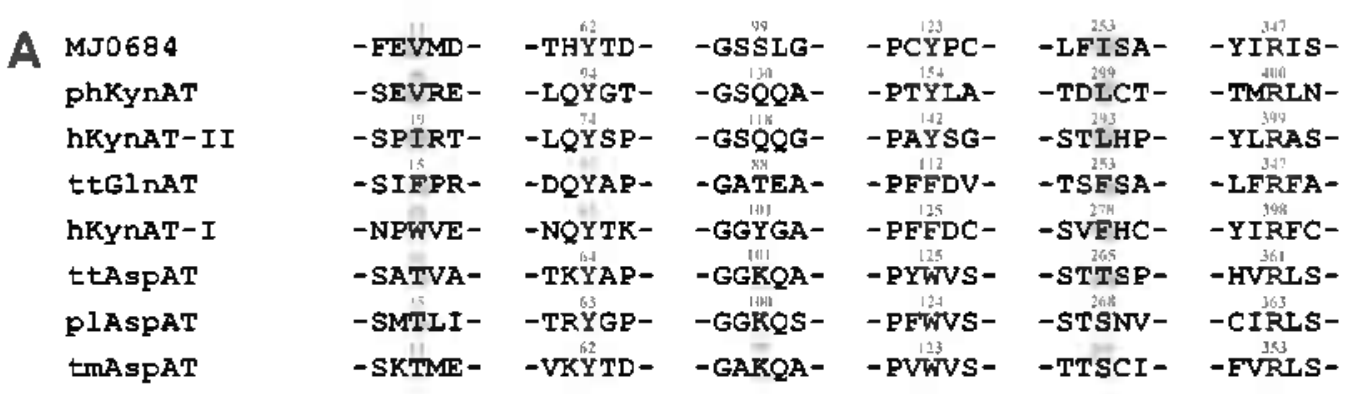

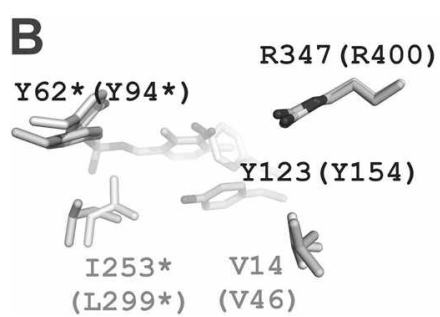

MJ0684 \& phKynAT

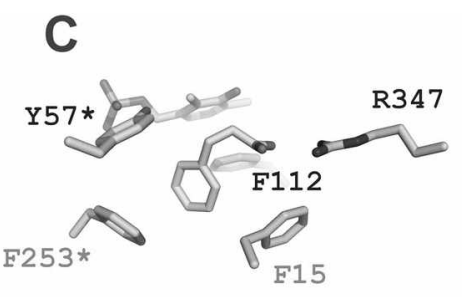

ttGlnAT

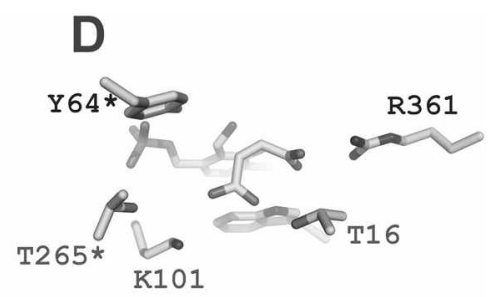

ttAspAT

Figure 2. (A) Stucture-based primary sequence alignment. MJ0684 is aligned with tamily I $\gamma$ KrnATs and AspATs for the key residues in substrate recognition and PLP binding. Residues in red and blue boses are critical for substrate recognition in KynATs and AspATs respectively. In cyan boses are the residues conserved commonly in KynATs and AspATs phKynAT is for KynAT from Pyrococcus horikoshii which is a bacterial homolog of human KynAT-II, hKynAT-II for human KYnAT-II, ttGlnAT for GlnAT trom Themus themophilus HB8 which is a bacterial homolog of human KynAT-I, hKynAT-I is for human KynAT, ttAspAT tor AspAT trom Themus themophilus HB8, plAspAT tor AspAT from Phomidium lapideum, and tmAspAT for AspAT from Themotoga mantima. (B) Superposition of M.T0684 and phKynAT. MJO684 (PDB code 2261) is colored in green and phKynAT (PDB code 1XoM) in light cyan. (C) ttGInAT in complex with 3-phenylpropionate (PDB code IV2F). (D) ttAspAT in complex with malate (PDB code IBKG). In (B)-(D), residues in red and blue are for the substrate recognition in KrnATs and AspATs respectively.

substrate aspartate. Notably MJ0684 also lacks the active site arginine residue conserved in the family I $\alpha$ AspATs for the same role (for example. R292* of pig cytosolic AspAT) ${ }^{7}$ In the active site of MJ0684, there is not found any basic residue which might be able to play the same role of the lysine residue in the family $\mathrm{I} \gamma$ AspATs or the arginine residue in the family I $\alpha$ AspATs. So the absence of the key residue for recognizing the aspartate strongly raises the possibility that MJ0684 may prefer other substrates. for example aromatic amino acids such as Kýn. Phe. Ty̆r and Trp as BLAST and DALI search results commonly indicate as the secondly most probable substrates.

In addition to the absence of the lysine or arginine residue for recognizing the side chain carboxylic group of an aspartate. one additional important structural feature is observed in the active of MJ0684 regarding the substrate specificity. That is the presence of two hydrophobic residues conserved in KynATs, not in AspATs. for recognizing aromatic amino acids as substrates. In the crystal structure of the bacterial homolog of human KynAT-I (ttGlnAT: GlnAT from Thermus thermophihus HB8: PDB code lV2F) ${ }^{\hat{3}}$ for example, it has been shown that the phenyl group of the substrate is surrounded by F15. F253*. Y57* and F112 (Fig. 2C). Among those four residues. Y57* and F112 interact with the cofactor PLP also: the former hydrogen bonds to the oxygen in the phosphate group of PLP and the latter interacts with pyridoxal ring of PLP via $\pi-\pi$ stack. These two residues are strongly conserved through various kinds of
ATs regardless of the substrate specificity (Fig. 2A, B, C and D). So the major role for $\mathrm{Y}^{*}{ }^{*}$ and F112 may be to coordinate the cofactor. Therefore the key residues for recognizing aromatic amino acid substrates should be the other two. i.e. F15 and F253* (Fig. 2C). These two hydrophobic residues are conserved in other KynATs such as human KynAT-I (W18 \& F278*: PDB code 1W7M; Fig. 2A) ${ }^{2+}$ and a bacterial homolog of human KynAT-II (V46 \& L299*: PDB code 1X0M: KynAT from Pyrococcus horikoshii: Fig. 2A and B). ${ }^{35}$ Just as in the active site of these KynATs, MJ0684 also has hydrophobic residues in the same position which are V14 and 1253* (Fig. 2A and B). In contrast. AspATs do not have hydrophobic ones in equivalents positions. In ttAspAT, for example. those two residues are T16 and T265* which participate in recognizing the distal carboxylic group of the substrate (Fig. 2A and D). T16 directly forms a hydrogen bond with the distal carboxylic group and T265 forms a hydrogen bond with K101 which directly interact with the distal carboxylic group. These two threonine residues are conserved in other fanily I $\gamma$ AspATs such as AspAT from Phormidium lapidetm (plAspAT. PDB code $1 \mathrm{~J} 32)^{36}$ and tmAspAT ${ }^{19}$ (Fig. 2A). In summary, two residues in these positions may be the determinants of the substrate specificity. at least differentiating between KynATs and AspATs. It looks obvious from the observation that there are hydrophobic ones in those positions in KynATs. but threonine or serine in AspATs (Fig. 2A). Therefore. the presence of V14 and $1253^{*}$ in the same positions of MJ0684 strongly 
implies that MJ0684 would prefer aromatic amino acids to dicarboxylic amino acids as substrates, just as KynATs do. In addition. it is noteworthy that, anong two types of KynATs. MJ0684 resembles more KynAT-II than KynAT-I as shown in Figure 2A. 2B and 2C. Human KynAT-II and its bacterial homolog from Pyococcts horikoshii (phKynAT) have I19L293* and V46-L299* respectively just as MJ0684 has V14-I253* for substrate recognition (Fig. $2 \mathrm{~A}$ and $2 \mathrm{~B}$ ). Meanwhile. human KynAT-I and its bacterial homolog from Thermus thermophihus HB8 (ttGlnAT) have W18-F278* and F15-F253* in the same positions (Fig. 2A and 2C).

In conclusion, the crystal structure of MJ0684 reveals that it doesn't have any basic residue in the active site for recognizing the side chain carboxylic group of the aspartate as a putative substrate. Moreover MJ0684 has two hydrophobic active site residues conserved in the subfamily $\mathrm{I} \gamma$ KynATs. not in AspATs. for recognizing hydrophobic substrates. These two lines of structural observation in the active site strongly imply that MJ0684 would prefer hydrophobic amino acids to the aspartic acid as a substrate. MJ0684 is a novel example of AT whose overall structure and sequence are more sumilar to AspATs than any other ATs but the local structure of the active site is even more sinilar to KynATs than AspATs. However, it remains to be shown that the novel structural observation on MJ0684 may coniply with the actual substrate specificity determined by the enzymatic assay experiment.

Acknowledgments. The author thanks Dr. Se Won Suh for supporting this work in all aspects, Dr. Soo Hyun Eom for sharing X-ray machine, and Dr. Changsoo Chang. Dr. Seung-je Cho, Dr. Jae Young Lee for invaluable comments in the course of structure analysis. This work was supported by the Soongsil University Research Fund to JKY.

\section{References}

1. Hirotsu. K.: Goto. M: Okamoto. A.: Miyahara. I. Chem. Rec. $2005,5,160$.

2. Mehta, P. K.: Hale. T. I.: Christen, P. Eur. J. Biochem. 1993, 214. 549 .

3. Jensen. R. A.: Gu. W. J. Bacteriol 1996.178.2161.

4. Jager. J.: Moser. M.: Sauder. U.: Jansonius. J. N. J. Mol. Biol. 1994. 239.285

5. McPhalen, C. A.: Vincent. M. G.: Jansonilus. J. N. J. Mol. Biol. 1992, 225,495
6. Malashkevich. V. N.: Strohopytov. B. V: Borisov. V. V: Dauter. Z.: Wilson. K. S.: Torchinsky. Y. M. J. Mol. Biol. 1995. 247. 111.

7. Rhee. S.: Silva. M. M.: Hyde. C. C.: Rogers. P. H.: Metzler. C. M.: Metzler. D. E.: Arnone. A. J. Biol Chem. 1997, 272, 17293.

8. Jeffery. C. J; Barry: T; Doonan, S; Petsko. G. A; Ringe, D Protein Sci. 1998. 7, 1380.

9. Nakai. T.: Okada. K.: Akutsu. S.: Myyahara. I.: Kawaguchi. S.: Kato. R.: Kuramitsu. S.: Hirotsu. K. Biochemistry 1999. 38. 2413.

10. Altschul. S. F.: Madden. T. L.: Schaffer. A. A.: Zhang. T.: Zhang. Z; Miller, W. Lipman, D. J. Nucleic Acids Res. 1997, $25,3389$.

11. Yang. J. K : Chang. C; Cho. S. J; Lee. J. Y.: Yu, Y. G.; Eom. S. $\mathrm{H}$; Suh, S. W. Acta Coystallogr: D Biol. Crystallog: 2003, 59. 563.

12. Terwilliger. T. C. Methods Enzwnol. 2003. 37+. 22

13. Bricogne. G.: Vonrhein. C.: Flensburg. C.: Schiltz. M.: Paciorek. W. Acta Coystallogr: D Biol. Cyystallogr: 2003. 59, 2023.

14. Abrahauns, J. P.: Leslie, A. G. Acta Cinstallog: D Biol. Crystallog: 1996. 52. 30.

15. Jones. T. A.: Zou. T. Y.: Cowan. S. W: Kjeldgaard. M. Acta Crystallogr: A 1991. 47. 110 .

16. Brunger. A. T.: Adams. P. D.: Clore. G. M.: DeLano. W. L.: Gros. P. Grosse-Kunstleve. R. W. Jiang. J. S.; Kuszewski, J.: Nilges. M.; Pannu. N. S.; Read, R. J.: Rice, L. M.; Simonson. T.: Warren. G. L. Acta Constallogr: D Biol. Crustallog: 1998. 54, 905.

17. Laskowski. R. A.: MacArthur. M. W.: Moss. D. S.: Thornton. J. M. J. Appl Chestallogr: 1993. 26. 283.

18. Grishin. N. V.: Phillips. M. A.: Goldsmith. E. J. Protein Sci. 1995. 4. 1291.

19. Schwarzenbacher, R.: Jaroszewski. L:; von Delft. F.: Abdubek, P.: Ambing. E.: Biorac. T.; Brinen. L. S.: Canaves, J. M.; Cambell, J.; Chiu. H. T.: Dai. X.: Deacon. A. M.: DiDonato. M.: Elsliger. M. A.: Eshagi. S.: Floyd. R.: Godzik. A.: Grittini. C.: Grzechnik. S. K.: Hampton. E.: Karlak. C.: Klock. H. E.: Koesena. E.: Kovarik. J. S: Kreusch. A.; Kuhn, P.: Lesley. S. A.: Levin. I.; McMullan. D; MoPhillips, T. M.: Miller, M. D.; Morse. A.: Moy. K: Ouyang. J.: Page, R: Quijano. K: Robb. A.: Spraggon. G: Stevens. R. C.: van den Bedem. H.: Velasquez. T.: Vincent. J.: Wang. X.: West. B.: Wolf. G.: Xu. Q.: Hodgson. K. O.: Wooley. J.: Wilsont. I. A. Proteins 2004. 55. 759.

20. Blankenfeldt, W: Nowicki, C.: Montemartini-Kalisz, M.: Kalisz. H. M: Hecht, H. J. Protein Sci. 1999, 8, 2406.

21. Holm. L.: Sander, C. Methods Enzymol 1996. 266,653.

22. Hant. Q: Gao. Y. G.: Robinson. H.: Ding. H.: Wilson. S.: Li. J. FEBS J. 2005. 272.2198

23. Goto. M.: Omi. R.: Myahara. I.: Hosono. A.: Mizuguchi. H.: Hayashi, H.; Kagamivama, H.; Hirotsu, K. J. Biol. Chem. 2004. 279. 16518

24. Rossi, F, Han. Q; Li, J.: Li, J; Rizzi, M. J. Biol. Chem. 2004. 279.50214 .

25. Chon. H.: Matsumura. H:: Koga. Y: Takan1o. K: Kan1aya. S Proteins 2005. 61.685.

26. Kim. H.: Nakaoka. M: Yagi, M.: Ashida. H.: Hamada, K: Shibata, H.: Sawa. Y. J. Biosci. Biong. 2003. 95. 421 